\title{
Effect of Concentration on the Viscosity of Dilute Solutions"
}

\author{
By Robert Simha
}

\begin{abstract}
A theory of the higher coefficients of the viscosity-concentration curve of a suspension of nonspherical particles is presented. Starting with the simple model of a dumbbell, the flow around a single particle and its modification due to interaction with other particles are considered. It is shown that the coefficients $a_{i}$ in the equation
\end{abstract}

$$
\eta=\eta_{0}\left(1+a_{1} c+a_{2} c^{2}+a_{3} c^{3}+\ldots\right)
$$

are related to each other, namely,

$$
a_{2}=k_{1} a_{1}^{2}, a_{3}=k_{2} a_{1}^{3} \ldots,
$$

where the k's are independent of molecular size, in agreement with empirical equations. An explicit value for $k_{1}$ is obtained that applies also to models consisting of various arrays of spheres. The variation of $k_{1}$ with molecular shape is discussed, and various factors affecting the numerical values and the validity of the last-mentioned equation are pointed out.

\section{Introduction}

The dependence of the viscosity $\eta$ of a dilute solution on the concentration $c$ (expressed as volume fraction or as weight by volume) can be represented as a power series of the type

$$
\eta=\eta_{0}\left(1+a_{1} c+a_{2} c^{2}+a_{3} c^{3}+\ldots\right),
$$

if the solute is a nonelectrolyte. $\eta_{0}$ is the viscosity of the pure solvent. Considerable experimental and theoretical effort has been devoted to a determination of the coefficient $a_{1}$, the so-called intrinsic viscosity, for suspensions of particles of various shapes since Einstein's $[1]^{1}$ original hydrodynamic treatment of spheres. In these hydrodynamic theories, $a_{1}$ is a measure of the disturbance of the flow of the solvent due to the presence of the solute particles at infinite dilution. The total effect under such conditions is the sum of the effects of the individual particles. For the range of higher concentrations, a number of

\footnotetext{
1 Figures in brackets indicate the literature references at the end of this paper.

*A warded an A. Cressy Morrison Prize in Natural Science in 1948 by the New York Academy of Sciences.
}

empirical equations have been proposed. They can all be expressed as a power series of type given in eq 1. As one can readily see, the theoretical calculation of the higher coefficients $a_{2}, a_{3}$, etc., becomes increasingly complicated. It has been carried out explicitly first for the coefficient $a_{2}$ of a spherical suspension by the author [2]. $a_{2}$, a number independent of size for spheres, measures the disturbance of the flow around a particle by a second one at a finite distance from the first. The method applied in the particular case is analogous to the procedure first developed by Smoluchowski [3] to obtain the modification of Stokes' law for the resistance of a sphere caused by the presence of a second one. Smoluchowski's results, of which we shall make use, are obtained by a perturbation in terms of inverse powers of the distance between the two spheres.

Empirically it has been suggested $[4,5]$ that the coefficients $a_{1}$ and $a_{2}$ are related in polymer solutions by the equation

$$
a_{2}=k a_{1}^{2},
$$

where the factor $k$ is independent of the dimensions 
of the solute particle but may vary with the solute-solvent system. In several instances, although not always, this rule seems to apply satisfactorily. It is the purpose of this paper to examine the validity of the proportionality (eq 2), using first the simplest model for a nonspherical particle, namely, that of a dumbbell considered originally by Kuhn in his treatment of intrinsic viscosity [6]. This model can be extended to that of a pearl string currently used by various authors in the description of hydrodynamic properties of chain molecules.

In section II the velocity distribution around a single particle and the intrinsic viscosity $a_{1}$ are obtained because the results are needed for what follows. In sections III and IV, interaction effects between solute molecules are considered. In section $\mathrm{V}$ attention is devoted to the nature of the approximations used and to the effect of various factors on the validity of eq 2 and the magnitude of the parameter $k$.

\section{Flow around an isolated particle. Intrinsic viscosity}

Figure 1 illustrates the notations and reference axes used. $2 L$ is the length of the dumbbell and $a$ the radius. It will be assumed that $a / L \ll 1$. Let the flow of the pure solvent be represented by a simple shearing motion in the $X_{1} X_{2}$-plane, namely,

$$
u_{1}^{0}=q X_{2}, u_{2}^{0}=u_{3}^{0}=0,
$$

where $q$ is the velocity gradient and $u_{l}{ }^{0}$ the velocity component parallel to the $X_{l}$-axis. If the dumbbell is held in a given orientation relative to the reference system $X_{l}$, the centers $P$ and $Q$ have the coordinates $x_{k}(P)$ and $x_{k}(Q)=-x_{k}(P)$, respectively. Equation 3 then gives for the velocities at $P$ and $?$

$$
u_{1}(P)=q x_{2}(P) ; u_{1}(Q)=-u_{1}(P),
$$

where the $x_{k}(P)$ are functions of the length $L$ and the direction cosines of the particle axis. In the absence of rotatory Brownian motion, this flow will rotate the particle axis into preferred directions. If one superimposes upon the motion (eq 3) a second one resulting from the spins of the particle and considers the resulting force and torques acting on the spheres $P$ and $Q$, one arrives at a result corresponding in three dimensions to Kuhn's [6] and identical with Jeffery's [7] formulae

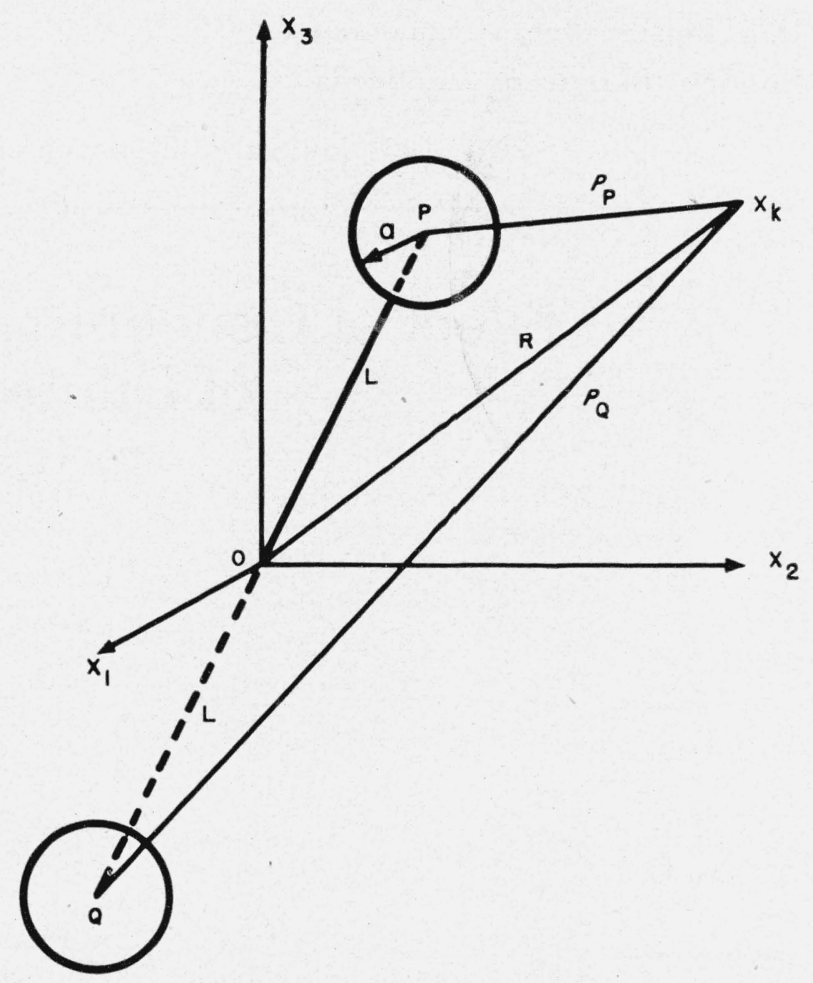

Figure 1. Dumbbell model and reference axes used and geometric relations involved.

for the rate of change of the orientation specialized for a thin rod. Here however we shall be concerned only with the case of so-called complete Brownian motion, where any orien tation tendency is absent. Following a previous procedure [8] we shall consider only the effects of the velocities (eq 3) and (eq 3a) on the particle.

The change in the unperturbed flow (eq 3) due to a single dumbbell is obtained as a solution of the hydrodynamic equations of motion, which neglecting inertia terms, assume in this case the form

$$
\left.\begin{array}{c}
\eta_{0} \nabla^{2} u_{l}=\frac{\partial p}{\partial X_{l}} \\
\frac{\partial u_{n_{b}}}{\partial X_{m}}=0 .
\end{array}\right\}
$$

Here, as in the following, summations from one to three are performed over twice occuring indices $m$. $p$ is the pressure. At infinite distance $R$, $u_{l}$ has to assume the form shown in eq 3 . If the velocity $u_{l}$ is transformed to a reference system $X_{l}(P)$ parallel to the first but with its origin at $P$, such that $X_{2}=X_{2}(P)+x_{2}(P)$, it is seen that 
the velocities $u_{l}$ consist of two parts if the mutual disturbance between the two spheres is neglected. The first satisfies the condition imposed by the $X_{2}(P)$-term. The required solution is of the type given by Einstein [1]. The second part, satisfying the constant velocity $q x_{2}(P)$ is of the type given by Stokes [9] for translational flow. Now for the calculation of the dissipated energy of the system particle plus solvent, it is sufficient to consider the velocity and pressure distribution at large distances compared with the dimensions of the solute molecule, and therefore a fortiori large compared with the radii $a$ of the spheres. As Einstein's solution contains terms of the order $a^{3} / \rho_{P}{ }^{2}$ (multiplied by a velocity gradient) and higher, while in Stokes' solution terms of the order $a / \rho_{P}$ (multiplied by a velocity) are found, the former can be neglected for our immediate purposes. Physically this amounts to concentrating the effect of each sphere on the solvent flow in its center $P$ or $Q$. The calculation of the perturbing field of flow then bears a certain formal similarity to that of the field of a rigid dipole, and including hydrodynamic interaction, to the calculation of dipole-dipole interaction. It should be remembered, however, that at distances close to the particle the equations given below are invalid. For the disturbance due to sphere $P$, we find in this manner from Stokes' solution neglecting terms of the order $a^{3} / \rho_{P}{ }^{3}$ :

$$
\left.\begin{array}{rl}
u_{l}{ }^{P} & =-\frac{3}{4} u_{1}(P) \frac{a}{\rho_{P}} \delta_{1 l}-\frac{3}{4} u_{1}(P) \frac{a X_{1}(P) X_{l}(P)}{\rho_{P}{ }^{3}} \\
p & =\text { const. }-\frac{3}{2} \eta_{0} u_{1}(P) a \frac{X_{1}(P)}{\rho_{P}{ }^{3}}
\end{array}\right\}
$$

where

$$
\left.\delta_{1 l}=\begin{array}{l}
1) \\
0
\end{array}\right\} \begin{aligned}
& l=1 \\
& l \neq 1
\end{aligned}
$$

The additional velocity $u_{l}^{Q}$ around sphere $Q$ is given by an analogous equation. From eq $3 \mathrm{a}$ and 5 it will be seen that the total velocity obtained by superposition of $u_{l}(P)$ and $u_{l}(Q)$ will contain terms

$$
\frac{1}{\rho_{P}}-\frac{1}{\rho_{Q}}
$$

and

$$
\frac{X_{1}(P) X_{l}(P)}{\rho_{P^{3}}}-\frac{X_{1}(Q) X_{l}(Q)}{\rho_{Q}^{3}}
$$

At points in the field of flow for which not only $\rho_{P}, \rho_{Q} \gg a$, but also $R \gg L$, it is permissible to expand these quantities in terms of the ratio $L / R$ (see fig. 1) and to discard the third and higher powers of $L$. Omitting details, one finds

$$
\left.\begin{array}{c}
\frac{1}{\rho_{P}}-\frac{1}{\rho_{Q}}=\frac{2}{R^{3}} \sum_{k} X_{k} x_{k}(P) \\
\frac{X_{1}(P) X_{l}(P)}{\rho_{P}{ }^{3}}-\frac{X_{1}(Q) X_{l}(Q)}{\rho_{Q}^{3}}= \\
\frac{6 X_{1} X_{l}}{R^{5}} \sum_{k} X_{k} x_{k}(P)-\frac{2}{R^{3}}\left[X_{1} x_{l}(P)+X_{l} x_{1}(P)\right] \\
\frac{X_{1}(P)}{\rho_{P^{3}}}-\frac{X_{1}(Q)}{\rho_{Q}{ }^{3}}=\frac{6 X_{1}}{R^{5}} \sum_{k} X_{k} x_{k}(P)-\frac{2 x_{1}(P)}{R^{3}} .
\end{array}\right\}
$$

The velocity to be added to eq 3 is then given by

$$
\left.\begin{array}{rl}
u_{l}=- & \frac{3}{2} \frac{u_{1}(P) a}{R^{3}}\left\{\sum X_{k} x_{k}(P) \delta_{1 l}-\right. \\
& \left.\left.\left[X_{1} x_{l}(P)+X_{l} x_{1}(P)\right]+\frac{3 X_{1} X_{l}}{R^{2}} \sum X_{k} x_{k}(P)\right\}\right\} \\
p=\text { const. }-3 \eta_{0} \frac{u_{1}(P) a}{R^{3}}\left[\frac{3 X_{1} \sum X_{k} x_{k}(P)}{R^{2}}-x_{1}(P)\right] \cdot
\end{array}\right\}
$$

Equation 5a is, of course, a solution of the equations of motion (eq 4). It is useful to write it in the form of Lamb's [9] general solution in terms of spherical harmonics. It is easily found that eq 5 a corresponds, using his notation, to:

$$
p_{-3}=p, \chi_{-2}=-\frac{3}{2} \frac{a u_{1}(P)}{R^{3}}\left[X_{2} x_{3}(P)-X_{3} x_{2}(P)\right] .
$$

In order to obtain the dissipation of energy caused by the flow (eq 5a), one considers a large spherical volume $V=\frac{4 \pi}{3} R_{0}{ }^{3}$ around the particle. Let $p_{r X_{l}}{ }^{0}$ and $p_{r X_{l}}$ denote the components of the radial stresses exerted per unit area on the surface of this sphere. Then one derives from eq 3 and the definition of the stresses:

$$
p_{r X_{1}}{ }^{0}=\eta_{0} q \frac{X_{2}}{R_{0}} ; p_{r X_{2}}{ }^{0}=\eta_{0} q \frac{X_{1}}{R_{0}} ; p_{r X_{3}}{ }^{0}=0
$$

Equation 5', together with an expression cited in [9] yield

$$
p_{r X}=\frac{18 \eta_{0} u_{1}(P) a}{R^{4}}\left\{\frac{2 X_{1}^{2}}{R^{2}} \sum X_{k} x_{k}(P)-X_{1} x_{1}(P)\right\}
$$


The rate of dissipation per unit volume is given by:

$$
\frac{d W}{d t}=\frac{1}{V} \int\left(p_{r X_{m}}{ }^{0}+p_{r X_{m}}\right)\left(u_{m}{ }^{0}+u_{m}\right) d S
$$

where the integral is extended over the surface of the sphere $R_{0}$. The result is, omitting terms of the order $1 / R_{0}$ and using eq. $3,3 \mathrm{a}, 3 \mathrm{~b}, 5 \mathrm{a}$, and 7 :

$$
\frac{d W}{d t}=\eta_{0} q^{2}\left[1+\frac{36 \pi}{5} \frac{a x_{2}(P)^{2}}{V}\right]
$$

if the particle is held in a fixed orientation. If the solution consists of $n$ particles dissolved in the volume $V$, such that $N=n / V$ represents their average number per unit volume, one has

$$
2 \frac{4 \pi}{3} a^{3} N=c
$$

where $c$ is the volume concentration. In the present case all effects are additive, and hence the dissipation of the whole solution is obtained by combining eq. $8 \mathrm{a}$ and 9 to

$$
\frac{d W}{d t}=\eta_{0} q^{2}\left(1+\frac{27}{10} \frac{x_{2}(P)^{2}}{a^{2}} c\right)
$$

In order to obtain the viscosity $\eta$ of the solution we follow the method of reference [1]. ${ }^{2}$ It will be assumed that the solute molecules are randomly distributed in the volume $V$. Let particle $i$ have its center situated at a point $\xi_{k}(i)$ in the $X_{k}$-system, $k=1,2,3 ; i=1,2, \ldots . n$. Its contribution $u_{l}(i)$ to the flow disturbance at a point $X_{k}$ is obtained from eq $5 \mathrm{a}$ if the coordinates $X_{k}$ are replaced by the relative coordinates $X_{k}-\xi_{k}(i)$. Hence we can write for the velocity distribution in the whole solution

$$
u_{l}=\delta_{1 \imath} q X_{2}+\sum_{i=1}^{n} u_{l}(i) .
$$

The viscosity of the pure solvent can be defined by the relation

$$
\left(\frac{d W}{d t}\right) \text { solvent }=\eta_{0} q^{2}=\eta_{0}\left[\frac{\partial u_{1}^{0}}{\partial X_{\Sigma}}+\frac{\partial u_{2}^{0}}{\partial X_{1}}\right]_{X_{k}=0}^{2} .
$$

In the same manner we regard the solution as a liquid with effective viscosity $\eta$ subject to an effective gradient $q_{s}$ defined as

$$
\begin{aligned}
\frac{d W}{d t} & =\eta q_{s}^{2}=\eta\left[\frac{\partial u_{1}}{\partial X_{2}}+\frac{\partial u_{2}}{\partial X_{1}}\right]_{X_{k}=0}^{2} \\
& =\eta\left\{q+\sum_{i=1}^{n}\left[\frac{\partial u_{1}(i)}{\partial X_{2}}+\frac{\partial u_{2}(i)}{\partial X_{1}}\right]_{X_{k}=0}\right\}^{2}
\end{aligned}
$$

where the left-hand side is given by eq $8 \mathrm{~b}$. As $\partial / \partial X_{k}=-\partial / \partial \xi_{k}(i)$, the sum in eq 10 a may be replaced by

$$
\begin{aligned}
& -\sum_{i=1}^{n}\left[\frac{\partial u_{1}(i)}{\partial \xi_{2}(i)}+\frac{\partial u_{2}(i)}{\partial \xi_{1}(i)}\right]_{X_{k}=0} \doteq \\
& -N \int_{V}\left(\frac{\partial u_{1}}{\partial \xi_{2}}+\frac{\partial u_{2}}{\partial \xi_{1}}\right){ }_{X_{k}=0}
\end{aligned}
$$

if an integral is substituted for the sum. The integration is to be carried out over the whole volume $V$. The expression can be transformed into an integral over the surface $S$, namely,

$$
-N \int_{S}\left(u_{1} \frac{\xi_{2}}{R_{0}}+u_{2} \frac{\xi_{1}}{R_{0}}\right) \underset{X_{k}=0}{d S}
$$

Substitution from eq $5 \mathrm{a}$ then leads to the result:

$$
q_{s}=q\left(1-\frac{9}{10} \frac{x_{2}(P)^{2}}{a^{2}} c\right)
$$

Thus the viscosity $\eta$ of the solution follows from eq $10 \mathrm{a}$ as

$$
\eta=\eta_{0}\left[1+\frac{27}{10} \frac{x_{2}(P)^{2}}{a^{2}} c\right]\left[1-\frac{9}{10} \frac{x_{2}(P)^{2}}{a^{2}} c\right]^{-2}
$$

Equation 12 gives the correct viscosity up to linear terms in the concentration $c$. Averaging over-all (random) orientations, $\left\langle x_{2}(P)^{2}\right\rangle=\left(L^{2} / 3\right)$, we finally obtain

$$
\eta=\eta_{0}\left(1+\frac{3}{2} \frac{L^{2}}{a^{2}} c\right)
$$

The intrinsic viscosity for the model under consideration is proportional to the ratio $L^{2} / a^{2}$ in concordance with the familiar result obtained for infinitely thin ellipsoids or rods not subject to any orientation effects by the flow gradient.

The quadratic and higher terms in $c$ obtained from eq 12 have to be discarded as long as hydrodynamic interaction effects that give contributions of the same character and order of magnitude [2], are not taken into account. This has sometimes not been observed in the literature when Einstein's results have been extended to higher concentrations. However, it can be seen that the higher terms in eq 12 will, after averaging the powers of $x_{2}(P)$, contain successively higher powers

\footnotetext{
2 The procedure given in reference [7] can equally well be made use of when interaction effects are absent.
} 
of the ratio $L^{2} / a^{2}$, thus confirming the empirical relation (eq 2 ) which may now be written as

$$
a_{2}=k_{1} a_{1}^{2} ; a_{3}=k_{2} a_{1}^{3} \ldots,
$$

where the $k$ 's are, for the present model, pure numbers and independent of the dimensions of the molecule.

Equation 13 could, of course, have been derived in a much more straight-forward manner by considering the frictional resistance to the flow offered by each of the two spheres. The rate of dissipation arising in this way is given by the product of frictional force and relative velocity. If the latter quantity is taken from eq $3 \mathrm{a}$, we have by application of Stokes' law

$$
\frac{d W}{d t}=\eta_{0} q^{2}+6 \pi \eta_{0} a q^{2}\left[x_{2}(P)^{2}+x_{2}(Q)^{2}\right] N .
$$

Elimination of $N$ by means of eq 9 leads at once to eq 13.

\section{Interaction between two particles}

The influence of a second particle on the viscous flow around a first has been considered first by Smoluchowski [3] for spheres in translational motion, leading to a modification of Stokes' law. The effect so obtained is a function of the ratio between radii and mutual distance of the two spheres. More exact calculations are reviewed in Oseen's treatise [3]. Rotating spheres have also been treated [2] and calculations for nonspherical particles have been presented $[3,10]$. The principle of Smoluchowski's method consists in seeking to fulfill by successive "reflections" the hydrodynamic boundary conditions on the surfaces of all solid bodies involved. That is, additional flow distributions are superimposed on the previous flow, to compensate for the additional disturbance at the boundary between particle and fluid induced by the neighbors of the former. Thus the boundary conditions are fulfilled to successively higher degrees of approximation. The first approximation that is sufficient for our present pur- pose has been shown [3] to fulfill the boundary conditions not at each point of the surface, which would make it an exact solution, but on the average over the whole surface. The velocity distribution is in other words such that, if averaged over the surface of the sphere, it gives no relative motion between the sphere and the liquid at their boundary. The solution previously employed by the author [2] in the viscosity treatment is of the same degree of accuracy.

Smoluchowski's original equations refer to spheres moving in the same direction with equal speeds. His results are modified slightly if this equality does not hold. Let the position of the centers of two spheres be given by $\xi_{k}{ }^{(1)}$ and $\xi_{k}{ }^{(2)}$ in a coordinate system fixed in space, their mutual distance by $R_{12}$ and their respective velocities in the $\xi_{1}$-direction by $U_{1}{ }^{(1)}, U_{1}{ }^{(2)}$. At a point $\xi_{k}$ (fig. 2) the velocity and pressure distribution pro-

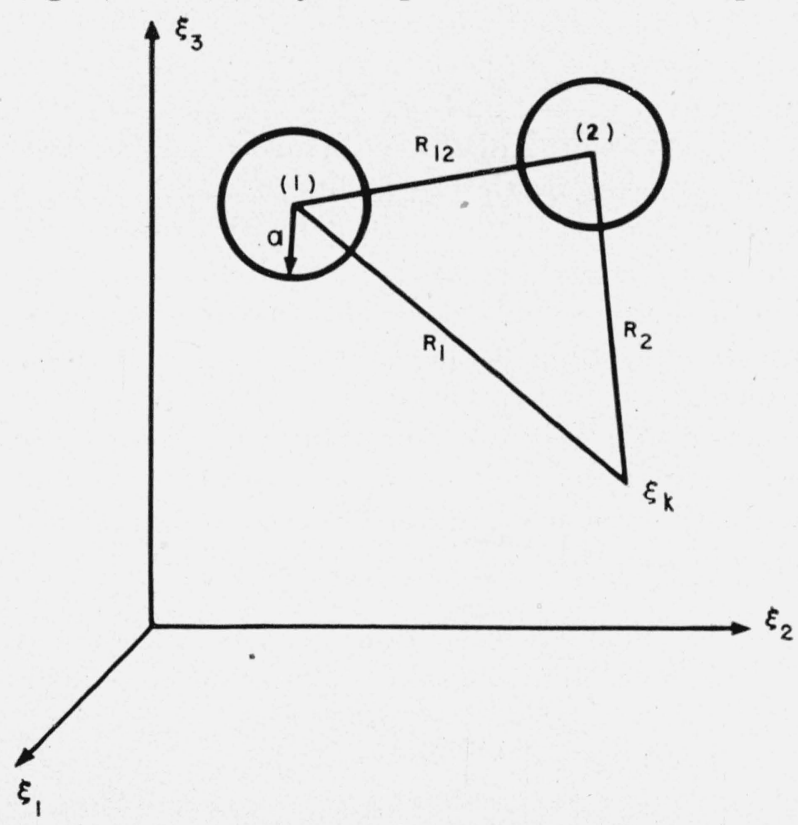

Figure 2. Geometric relations in interaction of two spheres, equations 14 and 15 .

duced by sphere 1 at large distances $R_{1}$ has the following form: ${ }^{3}$

where

$$
\left.\begin{array}{rl}
u_{l}{ }^{(1)} & =U_{1}{ }^{(1)} U_{l 1}{ }^{(1)}\left(\xi_{k}\right)-U_{1}^{(2)} U_{m 1}{ }^{(1)}\left(\xi_{k}{ }^{(2)}\right) U_{l m}{ }^{(1)}\left(\xi_{k}\right) \\
p^{(1)} & =\frac{3}{2} \eta_{0} a \frac{\left(\xi_{m}-\xi_{m}{ }^{(1)}\right)}{R_{1}{ }^{3}}\left[U_{1}{ }^{(1)} \delta_{m 1}-U_{1}{ }^{(2)} U_{m 1}{ }^{(1)}\left(\xi_{k}{ }^{(2)}\right]\right.
\end{array}\right\}
$$

\footnotetext{
${ }_{3}$ The author is indebted to E. O. Knox for checking eq 14 .
} 
$U_{l j}{ }^{(1)}\left(\xi_{k}{ }^{(2)}\right)$ represents this function at the point $\xi_{k}{ }^{(2)}$.

An analogous expression results for $u_{l}^{(2)}$ and $p^{(2)}$ It is seen that $u_{l}{ }^{(1)}$ and $p^{(1)}$ each consist of two terms. The first is, of course, Stokes' expression. The second one is a function of the-mutual distance and represents the interaction. Equation 14 leads to a frictional resistance

$$
\begin{aligned}
F_{l}^{(1)}= & 6 \pi \eta_{0} a\left[U_{1}^{(1)} \delta_{l 1}-\frac{3}{4} \frac{a}{R_{12}} U_{1}^{(2)} \delta_{l 1}-\right. \\
& \left.\frac{3}{4} \frac{a}{R_{12}{ }^{3}}\left(\xi_{1}{ }^{(2)}-\xi_{1}{ }^{(1)}\right)\left(\xi_{l}{ }^{(2)}-\xi_{l}{ }^{(1)}\right) U_{1}{ }^{(2)}\right]
\end{aligned}
$$

Thus the resultant resistance is no longer in the direction of motion.

Let us now consider a second dumbbell $(M, N)$ as shown in figure 3. Relative to its center the points $M$ and $N$ have the coordinates $x_{k}(M)$ and $-x_{k}(M)$, which are functions of the orientation of the axis $M N$ in respect to the $\mathrm{X}_{k}$-system. They determine $u_{1}(M)$ and $u_{1}(N)=-u_{1}(M)$, the respective velocities of the undisturbed solvent flow relative to the particle at points $M$ and $N$. As in section II we shall be concerned with distances $R$, which are large in comparison with $L$. Considering furthermore only molecules that are far apart, the modification of the Einstein-type of motion which gives rise to terms of the order $a^{6}$ [2], will be omitted and only $a^{2}$-terms retained. With these approximations one can make use of eq 14 and write the velocity around $P$ to be superimposed on $u_{l}^{P}$ in eq 5

$$
\begin{aligned}
\Delta u_{l}^{P}= & u_{1}(M) U_{m 1}{ }^{P}(M, P) \cdot U_{l m}^{P}(X, P)+ \\
& u_{1}(N) U_{m 1}{ }^{P}(N, P) U_{l m}{ }^{P}(X, P)
\end{aligned}
$$

with

$$
\begin{aligned}
& U_{l k}^{P}(X, P)=\frac{3}{4} \frac{a}{\rho_{P}}\left\{\delta_{l k}+\right. \\
& \left.\frac{\left[X_{l}-x_{l}(P)\right]\left[X_{k}-x_{k}(P)\right]}{\rho_{P}{ }^{2}}\right\} .
\end{aligned}
$$

$U_{l k}{ }^{P}(M, P)$ is obtained from the last equation by replacing $\rho_{P}$ by $\rho_{P M}$ and the coordinates $X_{j}$ by $\xi_{j}+x_{j}(M)$. The last term in eq 16 is obtained in a corresponding manner. An expression analogous to eq 16 results for $\Delta u_{l}{ }^{Q}$. We define the interaction coefficients $p_{j 1}$ and $q_{j 1}$ by means of the relations:

$$
\begin{aligned}
p_{j 1}= & U_{j 1}{ }^{P}(M, P)-U_{j 1}{ }^{P}(N, P) ; \quad q_{j 1}=U_{j 1}{ }^{Q}(M, Q)- \\
& U_{j 1}{ }^{Q}(N, Q)
\end{aligned}
$$

and obtain from eq 16 for the total velocity dis-" tribution around the molecule $P, Q$

$$
\begin{aligned}
u_{l}= & \left.u_{l} \text { (Stokes }\right)+u_{1}(M) p_{m 1} U_{l M}{ }^{P}(X, P)+ \\
& u_{1}(M) q_{m 1} U_{l m}{ }^{2}(X, Q)
\end{aligned}
$$

where the first term is given by eq 5a. The remainder contains functions of the mutual distance and orientation of the two interacting molecules. As stated previously, one sums over $m$ from one to three.

The quantities $U_{l m}{ }^{P}$ and $U_{l m}{ }^{Q}$ in eq 16a are of the same type as Stokes' velocity distribution and can be transformed in the same manner for large distances $R$. One finds in this manner in analogy to eq $5 \mathrm{a}$

$$
\begin{aligned}
& \left.u_{l}=u_{l} \text { (Stokes) }+\frac{3}{4} \frac{a u_{1}(M)}{R^{3}}\left(p_{m 1}-q_{m 1}\right)\left\{X_{k} X_{k}(P) \delta_{l m}-\left[X_{m} x_{l}(P)+X_{l} x_{m}(P)\right]+\frac{3 X_{l} X_{m}}{R^{2}} \sum_{k} X_{k} x_{k}(P)\right\}\right) \\
& p=p \text { (Stokes) }+\frac{3}{2} \eta_{0} \frac{a u_{1}(M)}{R^{3}}\left(p_{m 1}-q_{m 1}\right)\left\{\frac{3 X_{n}}{R^{2}} \sum X_{k} x_{k}(P)-x_{m}(P)\right\} .
\end{aligned}
$$

In analogy to eq 7 this makes a contribution to the $X_{1}$-component of the radial stress

$$
p_{r X 1}=p_{r X 1}(\text { Stokes })-\frac{9}{2} \eta_{0} a \frac{u_{1}(M)}{R^{4}}\left(p_{m 1}-q_{m 1}\right)\left\{\frac{4 X_{1} X_{m}}{R^{2}} \sum X_{k} x_{k}(P)-\left[X_{m} x_{1}(P)+X_{1} x_{m}(P)\right]\right\} .
$$

Application of eq 8 now yields for the total rate of dissipation per unit volume of the system consisting of solvent plus one particle $(P, Q)$ acted upon by a second one $(M, N)$ :

$$
\frac{d W}{d t}=\eta_{0} q^{2}\left\{1+\frac{36}{5} \frac{\pi a}{V} x_{2}(P)^{2}-\frac{6}{5} \frac{\pi a}{V} x_{2}(M)\left[3 x_{2}(P)\left(p_{11}-q_{11}\right)-2 x_{1}(P)\left(p_{21}-q_{21}\right)\right]\right\}
$$




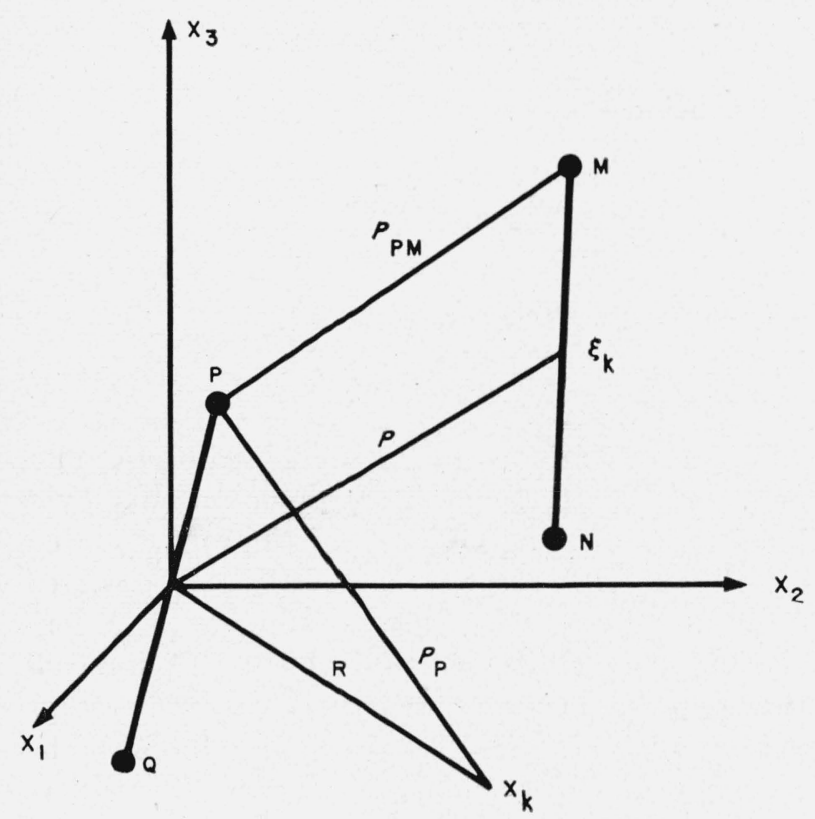

Figure 3. Geometric relations in interaction between two molecules, equation 16.

The interaction coefficients $p_{j 1}$ and $q_{j 1}$ can be evaluated for pairs sufficiently far apart in terms of the mutual distance $\rho$ (see fig. 3). A series expansion in terms of $1 / \rho$ gives, using eq 17 and discarding higher powers in $L / \rho$ :

$$
\begin{aligned}
& \left(p_{j 1}-q_{j 1}\right) \doteq-\frac{3 a}{\rho^{3}}\left\{\frac{15}{\rho^{4}} \xi_{1} \xi_{j} \sum \xi_{k} x_{k}(P) \cdot \sum \xi_{k} x_{k}(M)-\right. \\
& 3 \frac{\xi_{1} \xi_{j}}{\rho^{2}} \sum x_{k}(P) x_{k}(M)-\frac{3}{\rho^{2}}\left[\xi_{1} x_{j}(P)+\xi_{j} x_{1}(P)\right] . \\
& \sum \xi_{k} x_{k}(M)-\frac{3}{\rho^{2}}\left[\xi_{1} x_{j}(M)+\xi_{j} x_{1}(M)\right] \sum \xi_{k} x_{k}(P)+ \\
& x_{1}(P) x_{j}(M)+x_{j}(P) x_{1}(M)+\delta_{j 1}\left[\frac{3}{\rho^{2}} \sum \xi_{k} x_{k}(P) .\right. \\
& \left.\left.\sum \xi_{k} x_{k}(M)-\sum x_{k}(P) x_{k}(M)\right]\right\} .
\end{aligned}
$$

Equations $16 \mathrm{~b}$ and 20 constitute the complete solution of the interaction problem for two particles.

\section{Viscosity of Solution}

To obtain the quadratic concentration term in the viscosity one computes first from eq 19 the dissipation of the mixture. If we visualize again the centers of the solute molecules randomly distributed over the volume $V$, then for the ith particle $\left(P_{i} Q_{i}\right)$ situated at a point $\xi_{k}(i)$ and acted upon by a fixed particle $(M, N), p_{j 1}(i)-q_{j 1}(i)$ becomes a function of the relative coordinates $\xi_{k}-\xi_{k}(i)$. It is to be summed over all $\left(P_{i} Q_{i}\right)$ species interacting with one $(M, N)$ and then multiplied by the total number of particles. In this manner eq 19 yields

$$
\begin{aligned}
\frac{d W}{d t}= & \left(\frac{d W}{d t}\right)_{\text {stokes }}- \\
& \frac{9}{20} \eta_{0} q^{2} c \frac{x_{2}(M)}{a^{2}}\left\{3 x_{2}(P) \sum_{i}\left[p_{11}(i)-q_{11}(i)\right]-\right. \\
& \left.2 x_{1}(P) \sum_{i}\left[p_{21}(i)-q_{21}(i)\right]\right\}
\end{aligned}
$$

where the first term is given by relation (eq $8 b$ ). The summations may be replaced by integrations over the volume $V$. The use of the expression eq 20 in the whole region amounts to neglecting the influence of particles close to a given one. The error so committed increases, of course, at higher concentrations where the average distances between solute molecules become small. The two integrals can easily be transformed into surface integrals by means of Gauss' theorem. Omitting all terms that vanish on integration because of reasons of symmetry, one finds for instance, that the remainder in $\left(p_{21}-q_{21}\right)$ can be written as

$$
\begin{aligned}
\left(p_{21}-q_{21}\right)= & -\frac{3 a}{2}\left[x_{1}(P) x_{2}(M)+x_{2}(P) x_{1}(M)\right] \\
& {\left[\frac{\partial}{\partial \xi_{1}}\left(\frac{\xi_{1}}{\rho^{3}}-\frac{3 \xi_{1} \xi_{2}{ }^{2}}{\rho^{5}}\right)+\frac{\partial}{\partial \xi_{2}}\left(\frac{\xi_{2}}{\rho^{3}}-\frac{3 \xi_{1}{ }^{2} \xi_{2}}{\rho^{5}}\right)\right] }
\end{aligned}
$$

with a similar expression for $p_{11}-q_{11}$. The final result for the dissipation due to particles oriented in a given direction $x_{k}(P)$ interacting with particles in another direction $x_{k}(M)$ is

$$
\left.\begin{array}{rl}
\frac{d W}{d t}= & \eta_{0} q^{2}\left\{1+\frac{27}{10} \frac{x_{2}(P)^{2}}{a^{2}} c-\right. \\
& \frac{27}{50 a^{4}}\left[4 x_{1}(P) x_{2}(P) x_{1}(M) x_{2}(M)+\right. \\
& 6 x_{2}(P) x_{2}(M)\left(x_{2}(P) x_{2}(M)+\right. \\
& \left.\left.\left.x_{3}(P) x_{3}(M)\right)+x_{1}(P)^{2} x_{2}(M)^{2}\right] c^{2}\right\}
\end{array}\right\}
$$

The next step consists in calculating the effective velocity gradient $q_{s}$ defined in eq 10a. The velocity distribution around molecule $i$ in the presence of a perturbing molecule at an arbitrary point $\xi_{k}$ is derived from eq $16 \mathrm{~b}$ and 20 by replacing $X_{k}$ by $X_{k}-\xi_{k}(i)$ and $\xi_{k}$ by $\xi_{k}-\xi_{k}(i)$. 
The corresponding result for the whole mixture follows again from eq $5 \mathrm{~b} . q_{s}$ is then derived as in section II. The expression

$$
u_{1} \frac{\xi_{2}}{R_{0}}+u_{2} \frac{\xi_{1}}{R_{0}}
$$

to be integrated over the sphere $R_{0}$ now involves terms of the order $\frac{\xi_{k}{ }^{6}}{R_{0}{ }^{11}}$ and $\frac{\xi_{k}{ }^{4}}{R_{0}{ }^{9}}$. The integration requires the values of the following integrals over the surface of the spherical enclosure:

$$
\begin{aligned}
\int \xi_{1}{ }^{2} \xi_{2}{ }^{2} d S= & \frac{4 \pi}{15} R_{0}{ }^{6} ; \quad \int \xi_{1}{ }^{4} \xi_{2}{ }^{2} d S= \\
& \frac{4 \pi}{35} R_{0}{ }^{8} ; \quad \int \xi_{1}{ }^{2} \xi_{2}{ }^{2} \xi_{3}{ }^{2} d S=\frac{4 \pi}{105} R_{0}{ }^{8} .
\end{aligned}
$$

The integrand written above depends upon the mutual orientation in each molecule pair. On averaging independently the $x_{k}(P)$ and $x_{k}(M)$ terms, there remains after a lengthy but straight forward calculation, omitting all terms which vanish for random orientation:

$$
\begin{aligned}
& n N \mathcal{S}\left(u_{1} \frac{\xi_{2}}{R_{0}}+u_{2} \frac{\xi_{1}}{R_{0}}\right) d S= \\
& n N \frac{162}{5} \pi q \frac{a^{2}}{R_{0}{ }^{3}} x_{1}(P)^{2} x_{2}(M)^{2} .
\end{aligned}
$$

Instead of eq 12, one finds now, using eq $19 \mathrm{~b}$, that

$$
\eta=\eta_{0} \frac{\left[1+\frac{27}{10} \frac{x_{2}(P)^{2}}{a^{2}} c-\frac{27}{10} \cdot \frac{7}{5} \frac{x_{2}(P)^{2} x_{2}(M)^{2} c^{2}}{a^{4}}\right]}{\left[1-\frac{9}{10} \frac{x_{2}(P)^{2}}{a^{2}} c-\frac{9}{10} \cdot \frac{27}{4} \frac{x_{2}(P)^{2} x_{2}(M)^{2}}{a^{4}} c^{2}\right]^{2},}
$$

where the denominator represents $q_{s}{ }^{2}$. Expanding up to quadratic terms in the concentration and averaging over the direction cosines, we arrive at the following result for the viscosity of the solution:

$$
\eta=\eta_{0}\left(1+\frac{3}{2} \frac{L^{2}}{a^{2}} c+\frac{87}{50} \frac{L^{4}}{a^{4}} c^{2}\right) .
$$

On the other hand, eq 12 would have given for the coefficient of the quadratic term the smaller value $81 / 100$.

\section{Discussion}

Equation 13a yields for the coefficient $k_{1}$ defined in $2 \mathrm{a}$ the value

$$
k_{1}=0.77 \text {. }
$$

Had interaction effects not been taken into consideration, eq 12 would have given

$$
k_{1}=0.36 \text {. }
$$

These results refer so far to the model of a dumbbell. Let the molecule be represented next by a linear array of length $2 L$, consisting of a number of spheres with radii $a, \nu$ per unit length, arranged evenly. The simple considerations at the conclusion of section II, which lead to eq 13, indicate that now the factor $L^{2}$ is replaced by an average value $\left\langle L^{2}\right\rangle<L^{2}$. This is seen as follows: The contribution to the dissipated energy by a sphere located at a distance $y$ from the center of the molecule is proportional to $y^{2}$. This is to be summed over all groups in the molecule and multiplied by $N$, the number of molecules in unit volume. $N$ is inversely proportional to $2 \nu L$. Thus the intrinsic viscosity $a_{1}$ becomes proportional to

$$
\frac{2 \nu}{2 \nu L} \int_{0}^{L} y^{2} d y=\left\langle L^{2}\right\rangle
$$

In the case of a randomly coiled chain one averages over all internal configurations of the chain in addition to averaging over all orientations of the whole molecule. As long as coiling is not extensive the determining quantity has been shown repeatedly to be the mean square separation of chain ends. Reverting to the linear array, it can be seen from eq 19 and 20 that the interaction of parts of two molecules at distances $y_{1}$ and $y_{2}$ from their respective centers will contribute terms proportional to $y_{1}^{2} y_{2}^{2}$ to the dissipation rate. Consequently, the coefficient $a_{2}$ will be proportional to the square of $\left\langle L^{2}\right\rangle$ as one could have surmised. A corresponding result obtains for a solution consisting of moderately coiled chains. Thus the numerical values (eq 21 and 21a) should be valid also for a long extended molecule and a coil.

The value (eq 21) is larger than those derived experimentally from suitable extrapolations of viscosity-concentration curves, the latter being usually of the order of $0.3-0.7$ and more nearly comparable with the value (eq 21a). On the 
other hand, the theory in its present form can reproduce only approximately the contribution of the hydrodynamic interaction to the $k_{1}$ coefficient or rather to its excess over 0.36 . It has been stressed previously that the evaluation of the interaction coefficients $p_{j 1}, q_{j 1}$ (eq 17) as a series (eq 20) and therefore eq $19 \mathrm{~b}$ are valid only for large intermolecular distances. One could improve the situation by dividing the volume $V$ around the center of a molecule into three parts. The first comprises all particles with spherical groups so close to those of the center particle, that an expansion of the mutual disturbance in terms of the ratio $a / R_{12}$ (fig. 2) becomes altogether meaningless. In other words, solute molecules are practically touching each other whatever their relative orientation. From a hydrodynamic point of view, these complexes would be treated as single molecules. Such conditions, frequent at higher concentrations for large molecules will be disregarded here (see also below). In the second region intermolecular distances are large in comparison with the size of the individual group in the molecule, but are of the order of magnitude of the dimensions of the molecule. This would permit the application of Smoluchowski's equation (eq 14), but not of the expansion (eq 20). All further summations are to be performed in terms of individual group to group distances without using expansions in terms of molecular center to center distances. In the third region the current approximations are valid. Of course, the subdivision is somewhat arbitrary. The conditions in region two affect both the numerator and denominator in eq 12a. They should be more important in the calculation of the dissipated energy (see eq 19a) than in that of the effective velocity gradient $q_{s}$ (compare eq 10a), because of the direct summations over the $\left(p_{j 1}-q_{j 1}\right)$ occurring in the former. Also in this region a disturbance of the random mutual orientation of particles merely because of geometric reasons is possible.

The treatment in section II took no account of the hydrodynamic interaction between groups in the same molecule. For the dumbbell a simple calculation based on the result (eq 15) leads in a first approximation to a correction factor in the expression (eq 13) for $a_{1}$ equal to

$$
1+\frac{9}{20} \frac{a}{L}
$$

for $a / L \ll 1$. Also as a first approximation, $a_{2}$ remains unaltered. Thus only a decrease of $k_{1}$ by a few percent results from this effect. In an array of spheres the interaction of neighboring spheres with velocities of the same sign relative to the surrounding liquid will actually give a correction factor that is smaller than unity. The interaction between different groups within a strongly coiled molecule changes the flow pattern pronouncedly, as has been shown recently by several authors, and this influences also the total mutual disturbance between two different molecules.

It is perhaps noteworthy that the calculation in section II already gives as large a value of $k_{1}$ as 0.36. Effects other than purely hydrodynamic ones may contribute to the slope of the viscosityconcentration curve even at relatively moderate concentrations. Experimentally the reported initial slopes have sometimes been obtained by extrapolation at concentrations at which the conditions inherent in our derivation are no longer fulfilled; the solute molecules of large molecular weights are no longer sufficiently far apart on the average. There are indications in the literature that at concentrations roughly below $0.1 \mathrm{~g} / 100 \mathrm{ml}$ solutions of chain polymers exhibit steeper slopes than the extrapolated straight lines. Qualitatively similar effects have been observed in tobacco mosaic virus solutions [11]. In the latter case one is dealing with long rod-like particles and the possibility of aggregation is strongly increased at higher concentrations. Orientation effects are then relatively more important and this reduces the viscosity at higher concentrations. Systematic measurements of the viscosity of very dilute polymer solutions as function of velocity gradient and solvent character are desirable to decide the nature of the apparent downward curvature, found on approaching the region of high dilution.

It is obvious that the present considerations do not apply directly if the nature or the identity of the individual molecule changes with concentration. For instance if formation of aggregates occurs which can be expressed, say, by an equilibrium constant $K$ between double and single molecules it is seen that this makes an additional contribution to the coefficient $a_{2}$, namely,

$$
+K\left(a_{1}{ }^{(2)}-a_{1}\right)
$$

where $a_{1}^{(2)}-a_{1}$ is the difference between the intrinsic viscosity of double and single molecules. 
Thus the higher coefficients in the expansion (eq 1) cannot be found solely by hydrodynamic considerations and therefore eq $2 \mathrm{a}$ is then not valid. This is particularly the case when the effect of velocity gradient in concentrated solutions is to be examined. However, even in dilute solution the interaction is more affected by orientation than the intrinsic viscosity.

Finally the present results for asymmetric particles may be compared with those previously obtained for compact spheres. The author's method of calculation [2] leads to a value $a_{2}=14.1$ [10]. Einstein's results give a value of 4 for $a_{2}$. Thus, for spherical particles, the corresponding values are $k_{1}=2.26$ and 0.64 , respectively. It would be of interest to calculate the gradual increase in the $k_{1}$-values with increasing symmetry by considering ellipsoidal particles of finite thickness. Such results would be of value in the case of protein solutions and also of chain polymers. One should expect a parallelism between $k_{1}$ and other parameters characterizing shape such as the exponent in the modified Staudinger equation. Also branching and cross-linking should result in an increase of $k_{1}$. Such changes have been reported. It appears from the foregoing considerations that, leaving aside other factors, the parameter $k_{1}$ will remain independent of molecular weight as long as the degree of coiling does not change markedly.

\section{Conclusions}

In treating the concentration dependence of $\eta_{s p}$, two effects may be considered at low concentrations. Aggregation, or entanglements, and hydrodynamic interaction of distinct molecular entities. The first makes a contribution to the viscosity of the solution, which depends on the frequency of the aggregates and can be described in terms of the intrinsic viscosity of an aggregate. The second one leads to results that are in agreement with empirical relations proposed for dilute solutions. Without explicit calculation of the cubic and higher terms in $c$ it cannot be seen whether $\eta_{s p}$ is represented by the binomial formulas given by various authors. The present work indicates that they probably are not strictly valid.
The numerical value derived for $k_{1}$ (eq $2 \mathrm{a}$ and 21 ) is of the correct order of magnitude but somewhat larger than those encountered for solutions of chain molecules in good solvents, to which the model used is applicable under certain conditions. It seems to be more nearly in agreement with some numerical results for globular proteins. Possible experimental and theoretical reasons for this divergence have been outlined.

The parameter $k_{1}$ depends in a characteristic manner on the shape, as determined for coiling molecules by their structure, the solvent environment, and also on the molecular weight. This conclusion can be reached on the basis of the results obtained for two extreme cases treated here and in previous work, respectively. In this connection it is noteworthy that globular proteins appear to give larger values for $k_{1}$ than chain polymers. The region between the two extremes of an "open" coil, or dumbbell, and a sphere remains to be treated. On the basis of such a treatment it may become possible to derive information about the properties of the solute similar to that presently obtained from intrinsic viscosities.

It is of interest to examine the influence of the mutual disturbance on the viscosity at finite velocity gradients, when partial orientation in the field of flow occurs. Effects present at infinite dilution should be more pronounced.

\section{References}

[1] A. Einstein, Ann. Physik 19, 289 (1906); 34, 591 (1911).

[2] E. Guth and R. Simha, Kolloid-Zeits. 74, 266 (1936).

[3] M. Smoluchowski, Bul. Acad. Sci. de Cracovie 1A, 28 (1911); C. W. Oseen, Hydrodynamik, Akademische Verlagsgesellschaft (Leipzig, 1927).

[4] G. W. Schulz and F. Blaschke, J. prakt. Chem. 158, 130 (1941).

[5] M. L. Huggins, J. Am. Chem. Soc. 64, 2716 (1942).

[6] W. Kuhn, Z. physik. Chem. [A] 161, 1 (1932).

[7] G. B. Jeffery, Proc. Roy. Soc. [A] 102, 163 (1923).

[8] R. Simha, J. Phys. Chem. 44, 25 (1940).

[9] H. Lamb, Hydrodynamies (6th ed. p. 596 and 598, Dover Publications, New York, N. Y., 1945).

[10] O. Gold, Thesis (Vienna, 1937).

[11] H. K. Schachman, J. Am Chem. Soc. 69, 1841 (1947).

Washington, December 23, 1947. 